\title{
ESTUDIO BIBLIOMÉTRICO DE LA REVISTA ESPAÑOLA DE INVESTIGACIONES SOCIOLÓGICAS (1978-2002)
}

\author{
Pedro López López
}

Universidad Complutense, Escuela Universitaria de Biblioteconomía y Documentación

\section{Ángel Villagrá Rubio}

Consejo Superior de Investigaciones Científicas, CINDOC

\begin{abstract}
RESUMEN
Se presenta un estudio bibliométrico de la Revista Española de Investigaciones Sociológicas, con motivo de su $25 .^{\circ}$ aniversario. Los aspectos estudiados son producción, colaboración y materias. La reconocida importancia de esta revista en España convierte este estudio en altamente representativo de la literatura sociológica de este país. Asimismo, el estudio se completa con un apartado dedicado a evaluar su calidad como revista científica.
\end{abstract}

\section{INTRODUCCIÓN}

El objeto del presente trabajo es la realización de un análisis bibliométrico descriptivo de la Revista Española de Investigaciones Sociológicas (REIS de aquí en adelante) al completar los cien primeros números (este mismo artículo se incluye en el análisis), publicados a lo largo de los últimos veinticinco años. El estudio pretende también contribuir al análisis sociológico (desde el punto de vista de la Sociología de la Ciencia) e historiográfico de la Sociología española de estos veinticinco años a través de uno de sus principales vehículos de comunicación científica. Ello se hace partiendo de una metodología cuantitativa que atiende a facetas externas o sociales de la actividad científica.

Como es conocido, la metodología bibliométrica se ocupa de aplicar "métodos estadísticos y matemáticos dispuestos para definir los procesos de la comu- 
nicación escrita y la naturaleza y desarrollo de las disciplinas cientificas, mediante el recuento y análisis de las distintas facetas de dicha comunicación" (Pritchard, 1969), con diversas aplicaciones en las áreas de la documentación y la ciencia. En este último caso, los procedimientos bibliométricos son útiles a los llamados estudios sociales de la ciencia (enfoque externalista; ver Medina, 1982 y 1983, y Torres Alberó, 1994). En España, esta metodología fue introducida por J. M. López Piñero y M. L. Terrada a principios de los setenta, y durante estas tres últimas décadas ha dado lugar a una provechosa labor investigadora que ha aportado cientos de trabajos, en forma de artículos de revista, tesis doctorales y comunicaciones en congresos. Incluso diversos organismos han estudiado las actividades de investigación y desarrollo (I+D) a partir de estudios bibliométricos (véase, p. ej., Sánchez Nistal, 1998).

Son la Medicina y la Psicología las disciplinas que más han utilizado estos procedimientos en nuestro país. En esta última materia, Lascuráin, López López y González Uceda recogen 259 artículos publicados entre 1977 y 1996 y que utilizan técnicas bibliométricas. En ambas disciplinas también encontramos numerosas tesis doctorales bibliométricas, así como una importante aportación a la historia de ambas, que se hace patente en sus respectivos congresos (en el caso de Psicología, gran parte de las comunicaciones presentadas a congresos se canalizan a través de los que celebra anualmente la Sociedad Española de Historia de la Psicología; en Sociología, en cambio, aunque hay algunos trabajos, el modelo permanece infrautilizado).

Los sociólogos de la ciencia ven en la comunicación científica un elemento esencial sin el cual no podrían registrarse progresos en cualquier disciplina. Hasta tal punto tiene importancia este elemento que se ha llegado a proponer como definición formal de ciencia "aquello que se publica en los artículos científicos» (Price, 1980), considerando que un científico es una persona que «alguna vez ha contribuido a la redacción de un artículo semejante». Ziman (1968), después de refutar el enfoque filosófico tradicional sobre la ciencia, sostiene que la ciencia es conocimiento público, y que su meta es "un consenso de la opinión racional sobre el campo más vasto posible». Un consenso que sólo será posible si los miembros de la comunidad científica difunden sus investigaciones para que dicha comunidad las someta a una evaluación crítica.

Entre los canales formales de comunicación que utiliza la ciencia, la revista se ha erigido, sin duda, en el principal de ellos ${ }^{1}$. Esto justifica el interés de su estudio a la hora de investigar las características sociales de una disciplina cien-

${ }^{1}$ Esta consideración es indudablemente válida en el contexto de las llamadas ciencias duras. No obstante, debe ser atemperada en el contexto de las ciencias sociales, donde el artículo científico no tiene tanta preeminencia. Igualmente, hay que señalar que los investigadores que trabajan para la industria o para algunos sectores específicos (por ejemplo, Defensa) tienen una presión social inversa a los que trabajan en institutos de investigación públicos o en universidades. Si en este último caso hay una presión social para que publiquen, ya que esto va a constituir un criterio importante para su promoción, en el primero la presión opera en el sentido contrario, puesto que sus investigaciones exigen discreción. 
tífica, ya que sus miembros dejan plasmado su trabajo fundamentalmente en los artículos publicados en ella. De manera que cuando se estudia una revista que es importante dentro de una disciplina, tenemos acceso a una serie de indicadores de la actividad investigadora que se produce en esa disciplina. En el caso que nos ocupa, resulta que la Revista Española de Investigaciones Sociológicas es, como veremos, muy probablemente la principal de las publicaciones periódicas de Sociología en nuestro país, por lo que su análisis será de gran interés para el estudio de esta disciplina.

\section{BASES MATERIALES Y METODOLOGÍA}

Se ha partido de 795 artículos publicados a lo largo de los años 1978 a 2002 en las secciones Estudios, Documentos, Notas de Investigación, Notas y Debate. Se han excluido los trabajos publicados en las secciones Texto Clásico (ya que no se trata de trabajos originales) y Crítica de Libros (un tipo de trabajo que no se considera artículo científico).

Para la realización del trabajo se ha construido una base de datos con el programa de gestión documental KNOSYS. Los campos diseñados para el posterior tratamiento de los datos de interés contenidos en los 795 artículos han permitido la elaboración de las tablas y los gráficos que requiere un estudio de estas características.

A partir de los 795 artículos se ha realizado un análisis centrado en las tres facetas típicas de un estudio bibliométrico descriptivo: producción, colaboración y materias tratadas.

Cabe señalar que existe un precedente de este trabajo (López López y Escalada López, 1999) en el que se examinó la producción de la revista en sus dos primeras décadas (1978-1997).

\section{RESULTADOS}

\subsection{Producción Científica}

Se han contabilizado 795 artículos publicados a lo largo de los años 1978 a 2002 en las secciones Estudios, Documentos, Notas de Investigación, Notas y Debate. La media de artículos por año ha sido 31,8. Esta media no concuerda con la reflejada en la tabla 1 porque la tabla se ha obtenido partiendo de la base de datos ISOC. En nuestro caso, hemos sido más selectivos en los artículos que hemos incluido en nuestro análisis. La evolución temporal de la producción queda reflejada en el gráfico 1 . Como puede verse, hay oscilaciones importantes, pero la línea de tendencia señala un crecimiento muy suave a lo 
largo de las dos décadas. Se aprecian tres picos importantes en 1992, 1996 y 1998, que se deben a números monográficos. En 1992, Manuel Martín Serrano coordina un monográfico dedicado a cambio social y transformación de la comunicación. El número contiene 17 artículos, lo que eleva sensiblemente la producción de ese año. En 1996 encontramos otros dos números monográficos, curiosamente consecutivos. Es lógico pensar que el primero (n. ${ }^{\circ} 73$ ), dedicado a la sociología de la vejez, estaba programado, pero no el segundo (n. $\left.{ }^{\circ} 74\right)$, que se dedica in memoriam a José Luis López Aranguren. Estos dos números, con 12 y 14 artículos, respectivamente, también elevan la producción de 1996 muy por encima de la media. Igualmente, encontramos dos números monográficos en 1998. Uno de ellos, el 84, dedicado a Sociología del Arte, tiene 15 artículos, lo que eleva notablemente la producción de ese año.

\section{GRÁFICO 1}

\section{Evolución temporal de la producción}

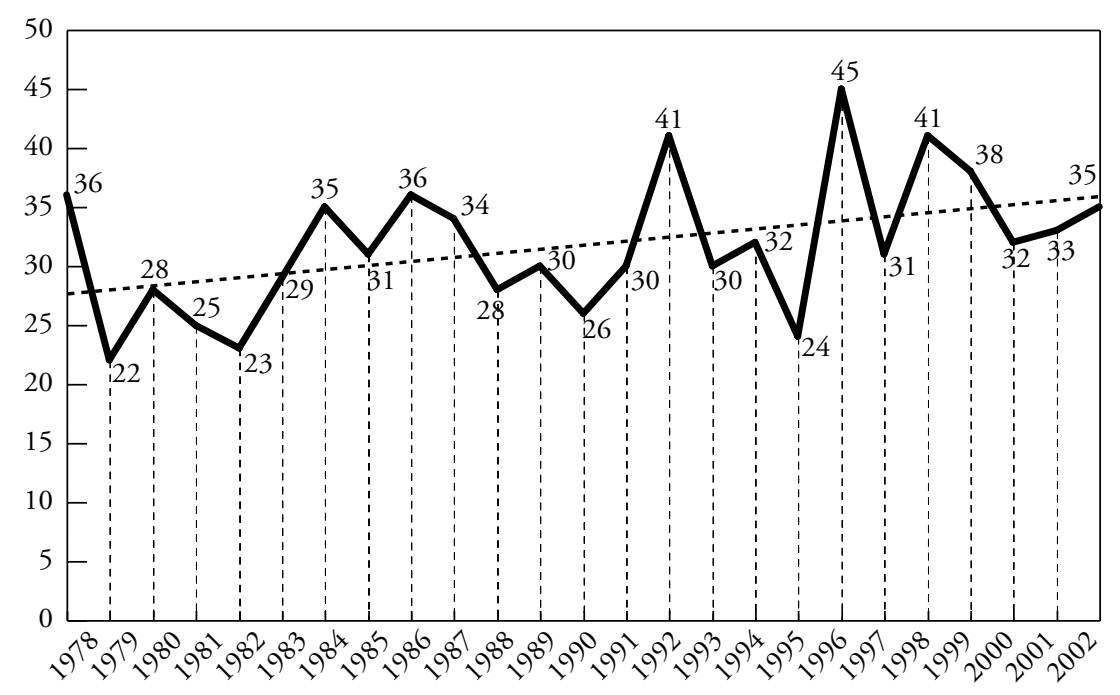

Si nos detenemos en una perspectiva comparativa, observamos que REIS se configura como revista nuclear de la Sociología española en estos veinticinco años. No sólo por la consistencia de su larga vida, sino también por el porte numérico de sus trabajos de investigación. Contemplando el ranking de producción de las principales revistas españolas de Sociología general (tabla 1), 
comprobamos que REIS ocupa la segunda posición en términos absolutos y relativos, justo por detrás de Documentación Social.

Pero a nadie se le oculta el diferente valor de los artículos de una y otra. Por su orientación general — Sociología aplicada - y su vocación de «intervención social», el carácter de los textos de Documentación Social se orienta más al estudio de casos, intercambio de experiencias, posicionamiento de diversos actores sociales, etc., que al análisis, teoría y metodología sociológicos propiamente dichos. Sus artículos se sitúan más en el plano de la comunicación informal y adolecen de menor interés científico general que los artículos de $R E I S$. Por ello, la comunidad sociológica valora mejor la formalización técnica y contenidos académicos de los artículos de REIS.

\section{TABLA 1}

Producción de las principales revistas españolas de Sociología (1978-2002)

\begin{tabular}{|c|c|c|c|}
\hline Revistas & $\begin{array}{l}\text { Número total } \\
\text { artículos }\end{array}$ & $\begin{array}{l}\text { Promedio } \\
\text { artículos/año }\end{array}$ & $\begin{array}{l}\text { Artículos totales } \\
\text { Sociologia (\%) }\end{array}$ \\
\hline Documentación Social (Madrid) .......................... & 1.335 & 54,8 & 6,41 \\
\hline $\begin{array}{l}\text { Revista Española de Investigaciones Sociológicas } \\
\quad \text { (Madrid) }\end{array}$ & $938^{*}$ & 37,4 & 4,50 \\
\hline Revista Internacional de Sociología (Córdoba) .. & 551 & 23,0 & 2,64 \\
\hline Papers. Revista de Sociología (Barcelona) ........... & 532 & 20,5 & 2,55 \\
\hline 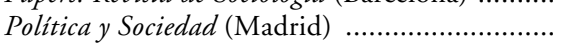 & 440 & 29,3 & 2,11 \\
\hline 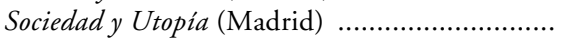 & 402 & 40,2 & 1,93 \\
\hline RS. Cuadernos de Realidades Sociales (Madrid) . & 381 & 14,6 & 1,82 \\
\hline 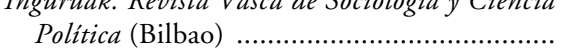 & 318 & 21,2 & 1,52 \\
\hline Revista Catalana de Sociología (Barcelona) ........ & 69 & 13,8 & 0,33 \\
\hline Arxius de Ciencias Sociales (Valencia) ............... & 51 & 10,2 & 0,24 \\
\hline 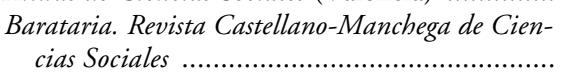 & 35 & 7,0 & 0,17 \\
\hline RIPS. Revista de Investigaciones Politicas y Socio- & & & \\
\hline $\begin{array}{l}\text { lógicas (Santiago de Compostela) ................ } \\
\text { RES. Revista Española de Sociología (Madrid) .. }\end{array}$ & $\begin{array}{r}18 \\
6\end{array}$ & $\begin{array}{r}18,0 \\
6,0\end{array}$ & $\begin{array}{l}0,08 \\
0,02\end{array}$ \\
\hline 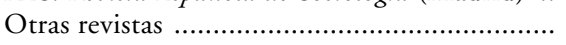 & 15.748 & 629,9 & 75,6 \\
\hline TOTAL artículos de revistas ....................... & 20.824 & 833 & \\
\hline
\end{tabular}

* Se incluyen, además de artículos originales, algunos textos que analizan resultados de encuestas, estudios sobre textos clásicos y algunas recensiones.

La tabla nos muestra, con todo, que el carácter nuclear de REIS, al que hemos aludido, tiene un valor bastante relativo, al menos en términos cuantitativos. En el conjunto de los 20.824 artículos de Sociología procedentes de 
revistas españolas que colecciona la Base de Datos ISOC-SOCIOLOGÍA (CSIC), el peso de REIS apenas representa el 4,5\% del total. Un porcentaje mayor, eso sí, que el de cualquier otra revista de Sociología general, salvo Documentación Social, pero realmente minúsculo en el conjunto de la producción sociológica española canalizada a través de revistas. Nótese que estos trece títulos de revistas, seleccionados entre los mejores, apenas rondan el $25 \%$ de todos los artículos que la Base de Datos ha clasificado como textos del Área de Conocimiento «Sociología». El restante $75 \%$ se dispersa enormemente en un largo listado de cerca de 100 títulos de revistas.

\section{Autoría}

Fijando la atención en la producción de los autores, constatamos que intervienen un total de 614 en los 795 trabajos. La distribución de estos autores según su número de firmas puede verse en la tabla 2.

TABLA 2

Distribución de autores según número de firmas

\begin{tabular}{|c|c|c|c|c|c|c|c|}
\hline & Firmas/autor & $\begin{array}{l}\text { Número } \\
\text { autores }\end{array}$ & Porcentaje & $\begin{array}{l}\text { Porcentaje } \\
\text { acumulado }\end{array}$ & $\begin{array}{l}\text { Número } \\
\text { firmas }\end{array}$ & Porcentaje & $\begin{array}{l}\text { Porcentaje } \\
\text { acumulado }\end{array}$ \\
\hline 14 & ......... & 1 & 0,16 & 0,16 & 14 & 1,46 & 1,46 \\
\hline 8 & n............ & 3 & 0,49 & 0,65 & 24 & 2,5 & 3,96 \\
\hline 7 & ......... & 7 & 1,14 & 1,79 & 49 & 5,1 & 9,06 \\
\hline 6 & ......... & 5 & 0,81 & 2,6 & 30 & 3,12 & 12,18 \\
\hline 5 & ......... & 12 & 1,95 & 4,55 & 60 & 6,24 & 18,42 \\
\hline 4 & …..................... & 17 & 2,77 & 7,32 & 68 & 7,07 & 25,49 \\
\hline 3 & ........................ & 30 & 4,89 & 12,21 & 90 & 9,37 & 34,86 \\
\hline 2 & ….................... & 87 & 14,17 & 26,38 & 174 & 18,11 & 52,97 \\
\hline \multirow[t]{2}{*}{1} & 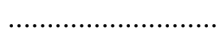 & 452 & 73,62 & 100 & 452 & 47,03 & 100 \\
\hline & TOTAL .............. & 614 & 100 & 100 & 961 & 100 & 100 \\
\hline
\end{tabular}

Como se observa en la tabla 2, casi tres cuartas partes de los autores $(73,62 \%)$ sólo hacen una contribución (una firma). El conjunto de firmas aportadas por este grupo asciende al 47,03\% del total. Por el otro extremo, tenemos una serie de autores productivos con al menos cinco firmas. Estos autores forman un grupo muy reducido (41 autores, 6,68\% del total) responsable de una cuarta parte de la producción (25,49\% de las firmas). Dentro de este grupo, el autor más productivo es Jesús M. de Miguel (Universidad Com- 
plutense), con 14 firmas. Por detrás aparecen tres autores con ocho firmas: Francisco Alvira (Univ. Complutense), Mauro F. Guillén (Universidades de Yale y Pennsylvania) y Manuel Martín Serrano (Univ. Complutense). La relación de los autores con más de cinco firmas es la siguiente:

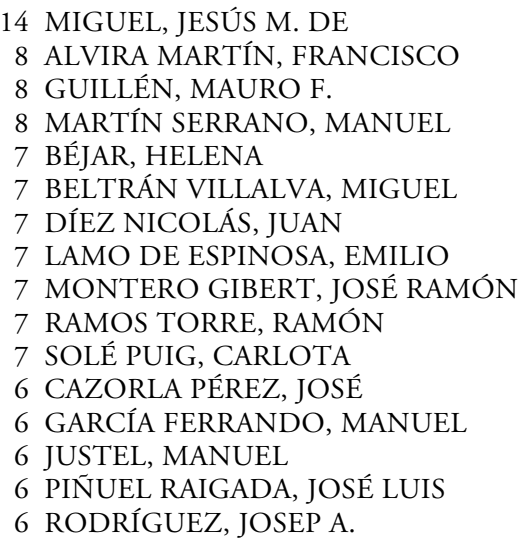

\author{
UNIV. BARCELONA \\ UNIV. COMPLUTENSE \\ UNIV. YALE / UNIV. PENNSYLVANIA \\ UNIV. COMPLUTENSE \\ UNIV. COMPLUTENSE \\ UNIV. AUTÓNOMA MADRID \\ UNIV. COMPLUTENSE \\ CSIC / UNIV. COMPLUTENSE \\ UNIV. CÁDIZ / UNIV. AUT. MADRID / CIS \\ UNIV. COMPLUTENSE \\ UNIV. AUTÓNOMA BARCELONA \\ UNIV. GRANADA \\ UNIV. AUT. MADRID / SIN AFILIACIÓN \\ UNIV. COMPLUTENSE \\ UNIV. COMPLUTENSE \\ UNIV. BARCELONA
}

De estos 16 autores, cinco firman siempre en solitario (Martín Serrano, Béjar, Beltrán, Ramos y Piñuel). Los demás firman normalmente en solitario, pero ocasionalmente colaboran con algún otro firmante. Sobre la colaboración nos extenderemos en el siguiente apartado.

\section{Participación institucional}

En cuanto a la participación institucional, se han identificado un total de 117 instituciones (tabla 3) ${ }^{2}$. Con diferencia sobre las demás, la Universidad Complutense destaca con 178 firmas. A continuación siguen la Universidad Autónoma de Barcelona (36 firmas), la Universidad Autónoma de Madrid (34), la de Barcelona (34) y la del País Vasco (31). Entre diez y veinte firmas aparecen la Universidad de Granada (27), el Centro de Investigaciones Sociológicas (21), el Consejo Superior de Investigaciones Científicas (19), la Universidad Pública de Navarra (12), la de Santiago (12) y la de Málaga (11).

Si desglosamos los centros de la Universidad Complutense, la Facultad de Ciencias Políticas y Sociología aparece en primer lugar, con 59 ocurrencias. El segundo centro es la Facultad de Ciencias de la Información, con 17 casos. Sin embargo, en 96 casos no consta el centro.

2 Las firmas que aparecen identificadas con su afiliación son 654 (un 68,05\% del total de firmas). En base a este número se dan los porcentajes. 
TABLA 3

Distribución de instituciones según número de firmas

\begin{tabular}{|c|c|c|c|c|c|c|}
\hline $\begin{array}{c}\text { Firmas/ } \\
\text { instituciones }\end{array}$ & $\begin{array}{l}\text { Número } \\
\text { instituciones }\end{array}$ & Porcentaje & $\begin{array}{l}\text { Porcentaje } \\
\text { acumulado }\end{array}$ & $\begin{array}{l}\text { Número } \\
\text { firmas }\end{array}$ & Porcentaje & $\begin{array}{l}\text { Porcentaje } \\
\text { acumulado }\end{array}$ \\
\hline 178 & 1 & 0,85 & 0,85 & 178 & 27,22 & 27,22 \\
\hline 36 & 1 & 0,85 & 1,70 & 36 & 5,5 & 32,72 \\
\hline 34 & 2 & 1,70 & 3,40 & 68 & 10,4 & 43,12 \\
\hline $31 \ldots \ldots \ldots \ldots \ldots \ldots$ & 1 & 0,85 & 5,95 & 31 & 4,74 & 47,86 \\
\hline $27 \ldots \ldots \ldots \ldots \ldots \ldots \ldots$ & 1 & 0,85 & 6,80 & 27 & 4,13 & 51,99 \\
\hline $21 \ldots \ldots \ldots \ldots \ldots \ldots \ldots$ & 1 & 0,85 & 7,65 & 21 & 3,21 & 55,2 \\
\hline 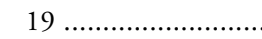 & 2 & 1,70 & 9,35 & 38 & 5,81 & 61,01 \\
\hline $12 \ldots \ldots \ldots \ldots \ldots \ldots \ldots \ldots$ & 2 & 1,70 & 11,05 & 24 & 3,67 & 64,68 \\
\hline $11 \ldots \ldots \ldots \ldots \ldots \ldots \ldots \ldots$ & 1 & 0,85 & 11,90 & 11 & 1,68 & 66,36 \\
\hline 9 …........................ & 2 & 1,70 & 13,60 & 18 & 2,75 & 69,11 \\
\hline 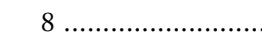 & 3 & 2,56 & 16,16 & 24 & 3,67 & 72,78 \\
\hline 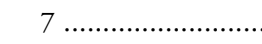 & 4 & 3,42 & 19,58 & 28 & 4,28 & 77,06 \\
\hline 6 …........................ & 2 & 1,70 & 21,28 & 12 & 1,84 & 78,90 \\
\hline $5 \ldots \ldots \ldots \ldots \ldots \ldots \ldots$ & 3 & 2,56 & 23,84 & 15 & 2,29 & 81,19 \\
\hline 4 .......................... & 1 & 0,85 & 24,69 & 4 & 0,61 & 81,79 \\
\hline 3 …...................... & 7 & 5,98 & 30,67 & 21 & 3,22 & 85,01 \\
\hline 2 ........................... & 15 & 12,82 & 43,49 & 30 & 4,59 & 89,60 \\
\hline 1 .......................... & 68 & 58,11 & 100,00 & 68 & 10,40 & 100,00 \\
\hline TOTAL ............... & 117 & & 100,00 & 654 & & 100,00 \\
\hline
\end{tabular}

\section{Origen geográfico de los artículos}

La producción según distribución geográfica queda reflejada en el gráfico 2. Para este dato se ha partido de la afiliación institucional de los autores. En el ámbito nacional $(87,48 \%$ del total) destacan claramente las instituciones ubicadas en Madrid. En segundo lugar aparece Cataluña (66 ocurrencias), seguida de Andalucía (40). En 70 ocasiones encontramos instituciones extranjeras $(12,52 \%$ del total).

Las instituciones que se han localizado en el extranjero (gráfico 3) pertenecen sobre todo a Estados Unidos (20 firmas). En segundo lugar aparecen Francia e Italia (13 firmas en ambos casos).

En la base de datos utilizada se ha recogido también el sexo de los autores, con lo que ha podido elaborarse el gráfico 4, que recoge porcentualmente la producción de hombres y mujeres. A lo largo de los veinticinco años se aprecia, con ayuda de las líneas de tendencia, cierto acercamiento, aunque llama la atención el comportamiento de los tres últimos años, que indica alejamiento. 


\section{GRÁFICO 2}

\section{Producción según distribución geográfica}

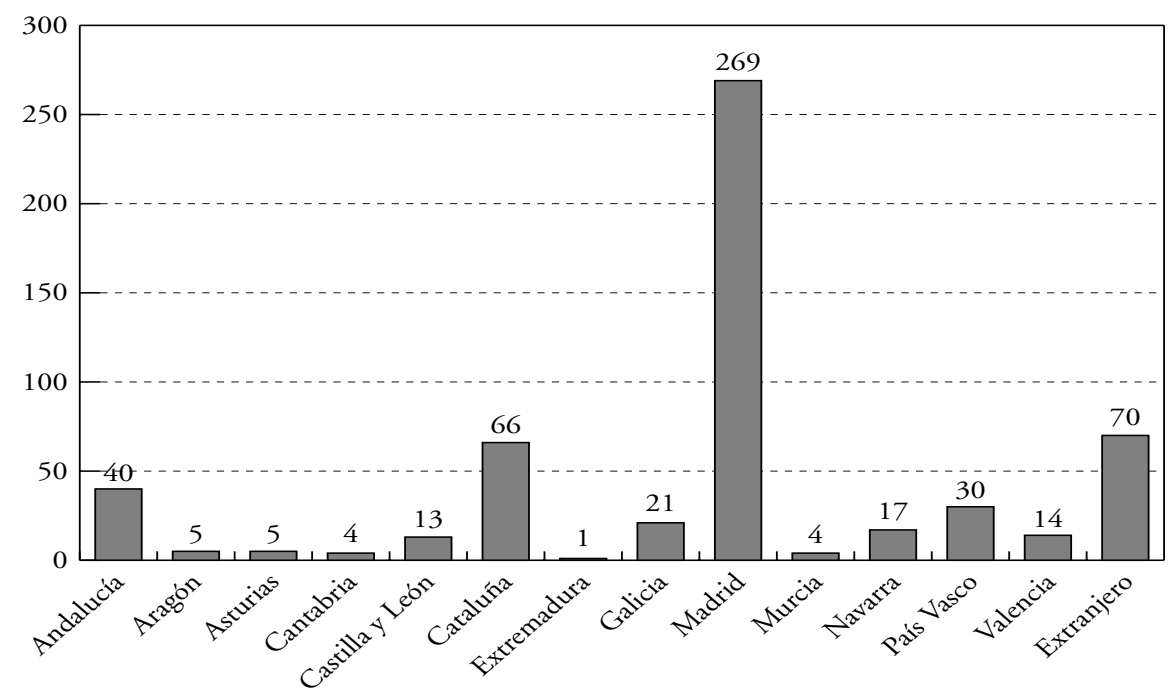

GRÁFICO 3

Producción ubicada en el extranjero

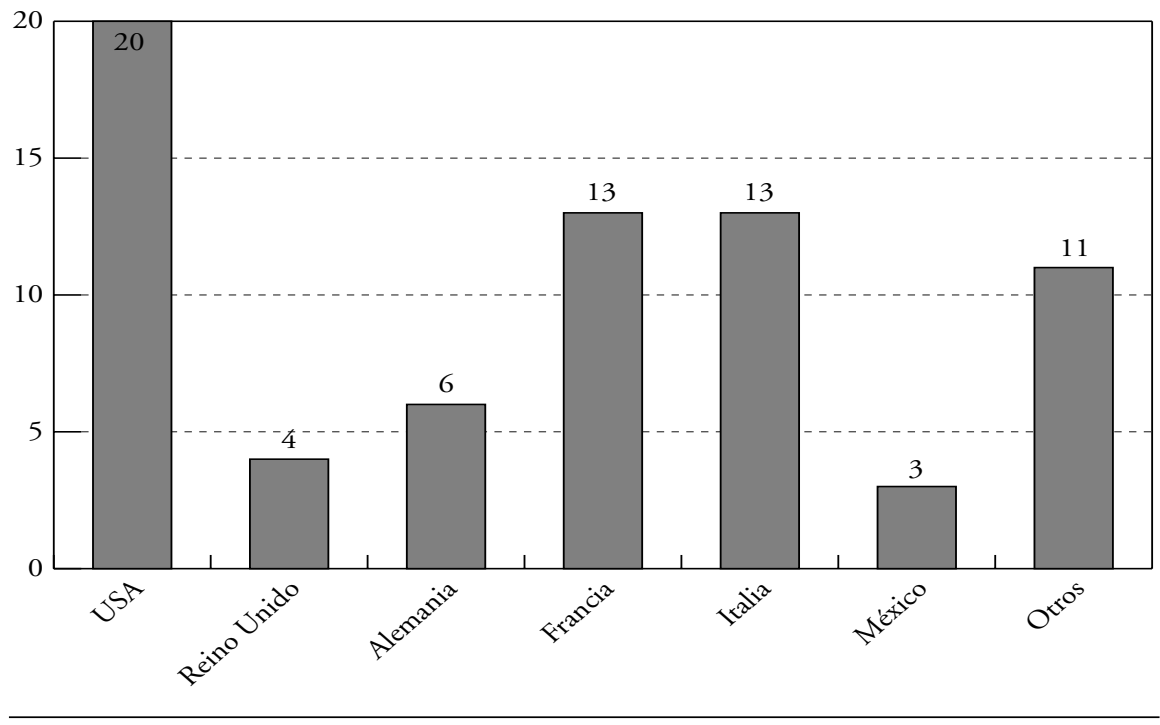




\section{GRÁFICO 4}

Evolución anual de la productividad por género (porcentajes)

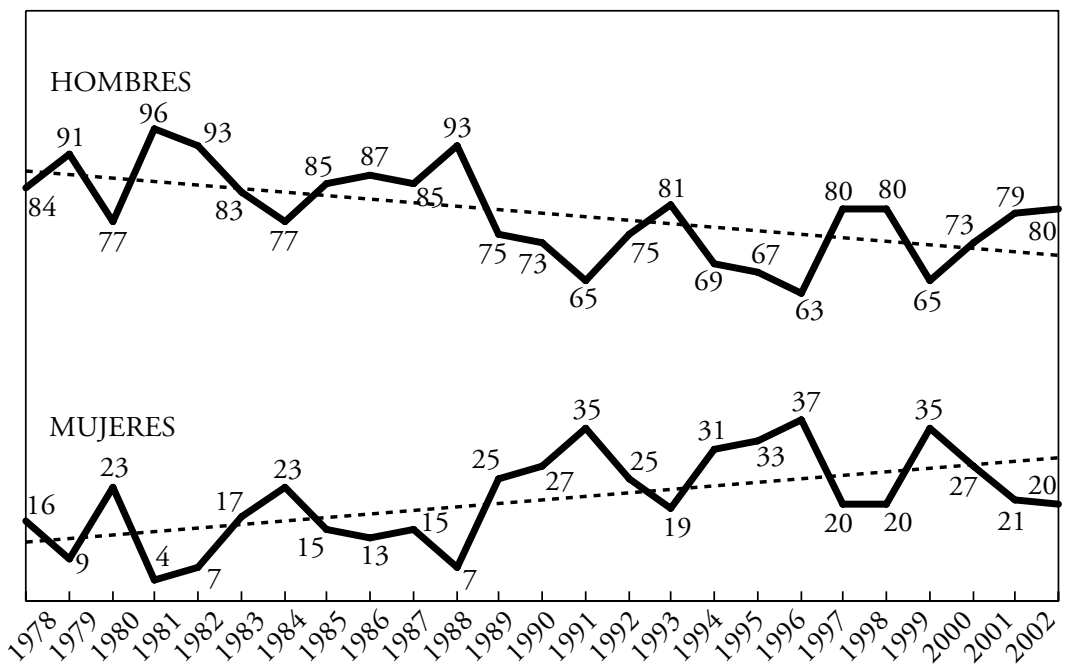

\subsection{COLABORACIÓN}

La colaboración entre autores (también entre instituciones y entre países) es uno de los aspectos que interesa a los sociólogos de la ciencia. Si a principios de siglo aproximadamente el $80 \%$ de los trabajos recogidos por el Chemical Abstracts aparecía firmado individualmente (Price, 1973), hoy la situación se ha invertido. La ciencia se hace cada vez más en colaboración. Dentro del contexto de las ciencias sociales, Berelson (1960) interpreta como madurez en la ciencia el paso de las disciplinas de palabras (que registran un bajo nivel de colaboración) a las disciplinas de datos (en las que la colaboración aumenta). Esta hipótesis relaciona claramente el progreso o madurez de una disciplina con un menor componente especulativo, lo que facilita el trabajo en colaboración. Existen otras hipótesis que relacionan el nivel de colaboración con el apoyo financiero recibido (mayores ayudas permitirían la formación de equipos de investigación y, por tanto, aumentarían el nivel de colaboración), como lo hacen Hirsch y Singleton (1965), estudiando precisamente la colaboración en revistas de Sociología entre 1934 y 1964 . Otra línea interpretativa la aporta Price (1963), al relacionar el aumento del nivel de colaboración con el paso de la Pequeña Ciencia a la Gran Ciencia. La primera es una ciencia artesanal donde interviene en mayor medida el investigador solitario, mientras que la segunda requiere en mayor medida la consolidación de equipos de investigación. También se han hecho estudios, aunque con datos contradictorios, rela- 
cionando el nivel de colaboración con la orientación básica o aplicada de las disciplinas.

En cualquier caso, hay que tener en cuenta que las diferencias entre las ciencias duras (empírico-experimentales) y las ciencias blandas (ciencias sociales y humanas) son manifiestamente apreciables. Algunas de estas últimas están claramente inclinadas al trabajo individual: Sociología, Ciencia Política, Filosofía, Derecho, Arte, Literatura, etc. Por ello, no sorprende que, al analizar REIS, en la distribución de artículos según número de firmas (tabla 4), nada menos que el $84,27 \%$ de los trabajos aparezcan firmados por un único autor. Es decir, sólo se registra un $15,73 \%$ de coautoría. Si contabilizamos los trabajos firmados por dos autores, vemos que añadimos otro $11,70 \%$, quedando en un insignificante $4,03 \%$ los trabajos que tienen tres o más firmas.

\section{TABLA 4}

Distribución de artículos según número de firmas

\begin{tabular}{|c|c|c|c|c|c|c|}
\hline Firmas/articulos & $\begin{array}{l}\text { Número } \\
\text { artículos }\end{array}$ & Porcentaje & $\begin{array}{l}\text { Porcentaje } \\
\text { acumulado }\end{array}$ & $\begin{array}{l}\text { Número } \\
\text { firmas }\end{array}$ & Porcentaje & $\begin{array}{l}\text { Porcentaje } \\
\text { acumulado }\end{array}$ \\
\hline ....... & 670 & 84,27 & 84,27 & 670 & 69,72 & 69,72 \\
\hline 2 ............................... & 93 & 11,7 & 95,97 & 186 & 19,35 & 89,07 \\
\hline 3 & 28 & 3,52 & 99,49 & 84 & 8,74 & 97,81 \\
\hline 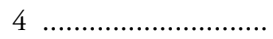 & 1 & 0,13 & 99,62 & 4 & 0,42 & 98,23 \\
\hline 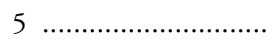 & 1 & 0,13 & 99,75 & 5 & 0,52 & 98,75 \\
\hline 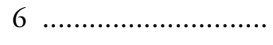 & 2 & 0,25 & 100 & 12 & 1,25 & 100 \\
\hline TOTAL ….............. & 795 & 100 & 100 & 961 & 100 & 100 \\
\hline
\end{tabular}

Sin embargo, si comparamos este porcentaje del 15,73\% de coautoría con otros datos del ámbito de las publicaciones sociológicas, resulta ser un valor privilegiado. Según un reciente estudio llevado a cabo en el CINDOC (CSIC) sobre la producción científica de la Comunidad de Madrid (cfr. Isabel Gómez Caridad, M. ${ }^{a}$ Teresa Fernández y Ángel Villagrá, Indicadores de $I+D$ en la Comunidad de Madrid, 2002) ${ }^{3}$ a partir del análisis de la información contenida en bases de datos nacionales e internacionales (que recogen, sobre todo, artículos de revistas), la tasa media de colaboración en el conjunto de las Ciencias Sociales en 1997-2000 es del 10,53\%, y la de las revistas de Sociología en su conjunto, del $6,42 \%$. La tabla 5 muestra el desigual comportamiento que en esta materia se produce en cada disciplina.

Puede apreciarse cómo la Sociología se encuentra muy lejos en materia de colaboración con respecto a la Psicología, la Arqueología o la Documentación,

${ }^{3}$ http://www.madridmasd.org/indicadores/produccion/produccionO.asp 


\section{TABLA 5}

Documentos en colaboración, por disciplinas (1999-2000)

\begin{tabular}{|c|c|c|c|}
\hline Disciplinas & $\begin{array}{c}\text { Artículos } \\
\text { en colaboración } \\
1997 / 2000\end{array}$ & $\begin{array}{l}\text { Artículos } \\
\text { totales } \\
1997 / 2000\end{array}$ & $\begin{array}{c}\text { Tasa } \\
\text { colaboración } \\
1997 / 2000\end{array}$ \\
\hline Psicología & 219 & 954 & 22,95 \\
\hline 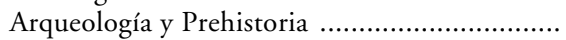 & 89 & 428 & 20,8 \\
\hline Biblioteconomía y Documentación .................. & 65 & 420 & 19,28 \\
\hline 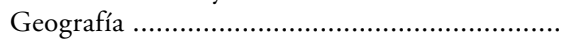 & 46 & 316 & 14,55 \\
\hline Economía & 369 & 3.146 & 8,55 \\
\hline 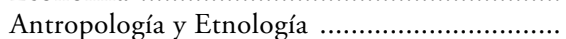 & 8 & 151 & 7,66 \\
\hline Lingüística & 25 & 327 & 7,64 \\
\hline 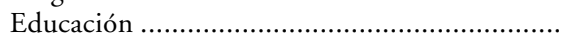 & 52 & 695 & 7,48 \\
\hline Sociologí & 65 & 1.012 & 6,42 \\
\hline Bellas Artes & 18 & 335 & 5,37 \\
\hline Estudios americanistas & 39 & 509 & 3,85 \\
\hline Historia & 33 & 1.012 & 3,26 \\
\hline 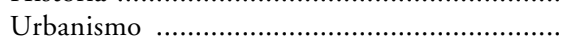 & 7 & 239 & 2,93 \\
\hline Derecho & 28 & 1.105 & 2,53 \\
\hline 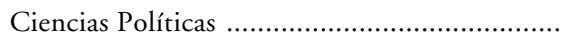 & 6 & 398 & 1,5 \\
\hline 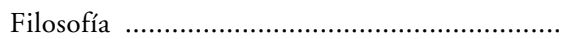 & 5 & 425 & 1,32 \\
\hline 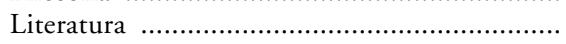 & 6 & 550 & 1,08 \\
\hline TOTALES & 1.080 & 12.022 & 8,98 \\
\hline
\end{tabular}

FUENTE: BDts. ISOC-Sociología.

y notablemente por encima de los trabajos de Ciencia Política, Filosofía o Literatura.

El indicador más utilizado para el nivel de colaboración es el número medio de firmas por trabajo (indice de coautoria). En REIS se registra una cifra muy baja: 1,21 . Es una media que sigue la pauta que apuntamos de baja colaboración en las ciencias sociales. Por ejemplo, según datos tomados de Extremeño (1998), los 10.115 artículos de Ciencia Política recogidos en la base de datos ECOSOC (CINDOC-CSIC) entre 1975 y mayo de 1998 registran una media de 1,14 firmas. Por su parte, A. Román (1993) al estudiar los artículos sobre la transición española en las revistas de ciencias sociales (internacionales y españolas) encuentra que un $86,35 \%$ aparecen firmados por un autor, y la media de firmas resultante es 1,19 . Y cuando toma un subconjunto de los artículos publicados exclusivamente en revistas españolas, el porcentaje de artículos con un solo firmante asciende al 95\%. Como se ve, las cifras son muy parecidas a las de nuestra tabla 4.

Para completar el punto dedicado al nivel de colaboración registrado, convie-

${ }^{4}$ Ver nota de la tabla 1. 
ne no sólo dejar reflejado el número medio de firmas de todo el período estudiado (1978-2002), sino analizar si esta media registra alguna variación contemplando el gráfico de la evolución anual del número medio de firmas por trabajo (gráfico 5). En él se aprecia un leve incremento al trazar una línea de tendencia. Este incremento tiene una interpretación indudablemente positiva. Gordon (1980) relaciona el mayor grado de colaboración con un mayor impacto de los trabajos, así como con una mayor calidad. También Bordons y cols. (1996) encuentran que los autores que colaboran con otros registran mayor productividad y visibilidad.

\section{GRÁFICO 5}

\section{Evolución anual del número medio de firmas}

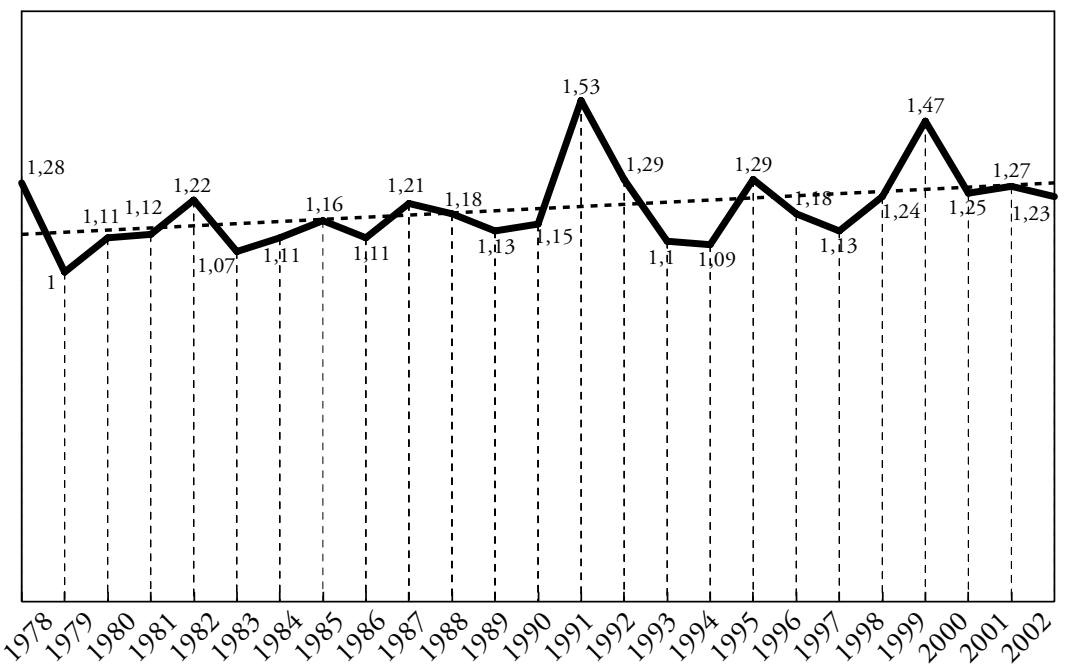

Por último, otro aspecto que interesa estudiar en el apartado de la colaboración es el referido a la estructura social de la misma. Esto se hace identificando los grupos de colaboración o colegios invisibles. Price y Beaver (1966) sugirieron detectar estos grupos a través de la autoría conjunta de artículos. Se parte de la consideración de que los autores que firman conjuntamente mantienen alguna vinculación entre sí. Metodológicamente, se procede detectando las redes de firmas entre autores que firman conjuntamente, sea directa o indirectamente (es decir, a través de colaboradores). Por tanto, la firma conjunta es el criterio de inclusión para la pertenencia a un grupo de colaboración. Ciertamente, el criterio es un tanto laxo cuando dos, o más, autores firman conjuntamente tan sólo un trabajo, situación que no refleja una colaboración permanente. Precisamente es esta situación la que hemos encontrado en la mayoría de los casos en nuestro trabajo. No obstante, es la vía metodológica que más 
frecuentemente se utiliza por evitar otros problemas metodológicos que aparecen de no seguirla. En este trabajo se ha utilizado, pero dejamos constancia de ello, al efecto de remarcar que el patrón de colaboración seguido por los autores de la REIS es ciertamente débil.

De los 614 autores que intervienen en la producción, sólo 268 (43,65\%) han escrito en coautoría alguna vez. Además, no se encuentra ningún grupo de colaboración numeroso, como puede apreciarse en la tabla 6.

\section{TABLA 6}

\section{Grupos de colaboración}

\begin{tabular}{|c|c|c|c|}
\hline & Autores/grupo & $\begin{array}{l}\text { Número grupos } \\
\text { con } \mathrm{n} \text { autores }\end{array}$ & Total autores \\
\hline 2 & 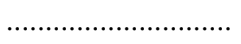 & 65 & 130 \\
\hline 3 & ............................ & 20 & 60 \\
\hline 4 & 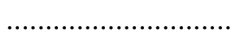 & 7 & 28 \\
\hline 5 & $\ldots \ldots \ldots \ldots \ldots \ldots \ldots$ & 1 & 5 \\
\hline \multirow[t]{2}{*}{6} & . & 5 & 30 \\
\hline & TOTAL .................. & 98 & 253 \\
\hline
\end{tabular}

Se han detectado 98 grupos de colaboración, que agrupan a menos de la mitad del total de autores, resultando que 346 autores (56,35\%) han escrito sus trabajos en solitario. El alto número de grupos corresponde a una estructura social muy atomizada, con una mayoría de grupos de tan sólo dos autores (65 grupos). El máximo de autores en un grupo es de seis. En este caso existen cinco grupos, encabezados por Jesús de Miguel (Univ. Barcelona), Francisco Alvira (Univ. Complutense), José Ramón Montero (Univ. de Cádiz, Univ. Autónoma de Madrid), Xavier Coller (Univ. Valencia, Univ. Barcelona) y Antonio Muñoz Carrión (Univ. Complutense). De estos grupos, el más productivo es el de José R. Montero, con 16 artículos que tratan cuestiones de Sociología Política. También es productivo el de Muñoz Carrión, con 12 artículos, y cuya materia principal es Sociología de la Comunicación.

\subsection{ANÁLISIS DE MATERIAS}

El análisis de materias se ha realizado partiendo de los códigos de la clasificación de la UNESCO asignados por los documentalistas del CINDOC, e introduciendo alguna pequeña adaptación conveniente a nuestras necesidades. Las categorías que han quedado son las siguientes: 
1) SOCIOLOGÍA POLÍTICA (incluye información y comunicación política).

2) CONFLICTOS INTERNACIONALES.

3) INVESTIGACIÓN Y DOCUMENTACIÓN SOCIOLÓGICA.

4) TEORÍA Y MÉTODOS DE LA SOCIOLOGÍA.

5) PSICOSOCIOLOGÍA.

6) CULTURA Y SOCIALIZACIÓN.

7) POBLACIÓN Y CAMBIO DEMOGRÁFICO.

8) ESTRUCTURA Y CAMBIO SOCIAL.

9) PROBLEMAS SOCIALES. POLÍTICA SOCIAL.

10) SOCIOLOGÍA DE LA CULTURA.

11) SOCIOLOGÍA URBANA Y RURAL.

12) SOCIOLOGÍA ECONÓMICA.

13) SOCIOLOGÍA DEL TRABAJO.

14) OCIO Y TIEMPO LIBRE.

15) SOCIOLOGÍA MILITAR.

El gráfico 6 muestra las frecuencias de cada una de estas categorías. Puede apreciarse que las materias más tratadas por los autores han sido «Sociología Política» (137 artículos), «Estructura y cambio social» (119 artículos) y «Teoría y Métodos de la Sociología» (98). Un comentario detallado al análisis de materias efectuado desborda las dimensiones de este trabajo.

\section{GRÁFICO 6}

\section{Análisis de materias}

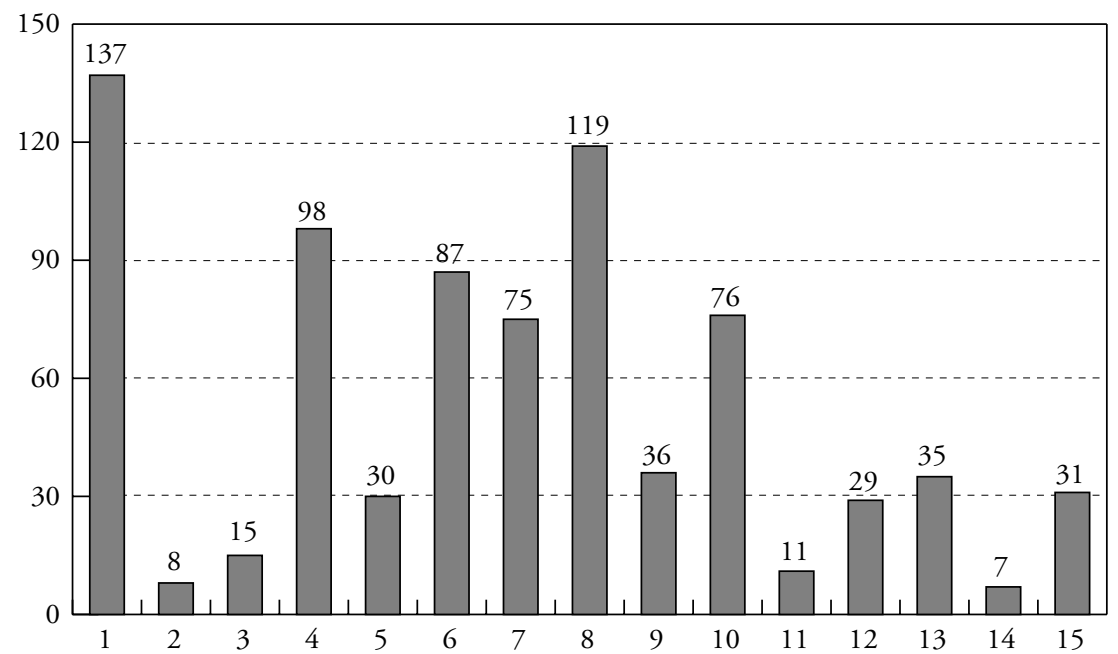




\section{CONCLUSIONES}

El trabajo realizado permite extraer algunas conclusiones de interés sobre una revista altamente representativa del quehacer de los sociólogos españoles en los últimos veinticinco años. En primer lugar, el estudio de la producción científica marca una pauta con numerosos picos hacia arriba y hacia abajo. Sin embargo, una línea de tendencia en el gráfico permite constatar un suave crecimiento a lo largo de todo el período. Cuando se observa la producción dentro de un contexto más amplio, puede concluirse que REIS es una revista nuclear de la Sociología española. En cuanto a los autores más productivos, han resultado ser Jesús M. de Miguel (Univ. Barcelona / Univ. Aut. Barcelona), Francisco Alvira (Univ. Complutense), Mauro F. Guillén (Univ. Yale / Univ. Pennsylvania) y Manuel Martín Serrano (Univ. Complutense). De los once autores más productivos (con siete firmas o más), seis pertenecen a la Universidad Complutense. La relevancia de esta Universidad queda corroborada al ser la institución que más veces aparece (178 ocurrencias), muy por delante de la segunda (Universidad Autónoma de Barcelona, 36 ocurrencias). También destacan las Universidades Autónoma de Madrid (34), la de Barcelona (34) y la del País Vasco (31).

En cuanto al patrón que sigue el trabajo en colaboración en esta revista, corresponde al del contexto científico al que pertenece, si bien el porcentaje de trabajos en coautoría es superior al registrado en el resto de trabajos de Sociología en la base de datos del CSIC. Por otro lado, a lo largo del período estudiado se constata un suave incremento en la evolución del número medio de firmas por trabajo. Los grupos de colaboración son muy numerosos, aunque predomina el trabajo individual (más de la mitad de los autores trabajan en solitario). El trabajo en colaboración suele ser de dos autores, no registrándose grupos de más de seis miembros.

El análisis de materias revela tres temáticas dominantes: «Sociología Política», "Estructura y cambio social» y «Teoría y métodos de Sociología».

Por último, cabe señalar que REIS es una revista de referencia en la comunidad sociológica española, y que un estudio de sus parámetros de calidad seguramente corroboraría esta impresión. Los autores de este trabajo disponen de algunos datos relativos al factor de impacto de la revista y a otros indicadores de calidad, datos que servirán para un próximo trabajo.

\section{REFERENCIAS BIBLIOGRÁFICAS}

Berelson, B. (1960): Graduate education in the United States, Nueva York, McGraw-Hill.

Bordons, M., y cols. (1996): «Local, domestic and international scientific collaboration in biomedical research", Scientometrics, vol. 37, n. 2 2, pp. 279-295.

Extremeño Placer, A. (1998): "Análisis cualitativo de la bbd Ecosoc», El Profesional de la Información, vol. $7, \mathrm{n} .^{\circ} 10$, pp. 4-11. 
Gordon, M. D. A. (1980): «Critical reassessment of inferred relations between multiple authorship, scientific collaboration, the production of papers and their acceptance for publication", Scientometrics, vol. 2, n. ${ }^{\circ}$ 3, pp. 193-201.

Hirsch, W., y Singleton, J. F. (1965): Research support, multiple autorship and publication in sociological journals 1934-1964. Preprint no publicado.

Lascuráin SÁnchez, M. L.; López López, P., y González UCeda, L. (1996): «Psicología y Bibliometría en España", Revista General de Información y Documentación, vol. 7, n. ${ }^{\circ}$ 2, pp. 37 46.

LÓpez López, P., y EsCalada López, C. (1999): «Veinte años de Sociología española a través de la Revista Española de Investigaciones Sociológicas (1978-1997)", Revista General de Información y Documentación, vol. 9, n. ${ }^{\circ}$ 1, pp. 161-173.

Medina, E. (1982): "Teorías y orientaciones de la Sociología de la Ciencia», Revista Española de Investigaciones Sociológicas, n. ${ }^{\circ} 20$, pp. 7-58.

- (1983): «La polémica internalismo/externalismo en la Historia y la Sociología de la Ciencia», Revista Española de Investigaciones Sociológicas, n. ${ }^{\circ} 23$, pp. 53-75.

Price, D. J. S. (1980): "Ciencia y tecnología: distinción e interrelaciones», en B. Barnes, Estudios sobre Sociología de la Ciencia, Madrid, Ed. Alianza (or.: 1972).

Price, D. J. S., y BeAver, D. (1966): "Collaboration in an invisible college», American Psychologist, vol. 21, pp. 1011-1019.

Pritchard, A. (1969): «Statistical Bibliography or Bibliometrics?», Journal of Documentation, vol. 25, n. ${ }^{\circ} 4$, pp. 348-349.

Román Román, A. (1993): Las revistas de Ciencias Sociales fuente para el estudio de la Historia: La transición (1975-1985), una perspectiva historiográfica y documental. Tesis doctoral presentada en la Universidad Complutense de Madrid.

RomÁn Román, A., y Alcain Partearroyo, M. a Dolores (2003): Indices de impacto de las revistas españolas de CC. Sociales, a partir del análisis de las revistas mejor valoradas por los Pares (Acción subvencionada MEC EA2002-0014). En prensa.

SÁnChez Nistal, J. M. (1998): «La producción científica de la Comunidad de Madrid en el trienio 1994-1996», en González Hermoso y De la Sota (coords.), Investigación y desarrollo en la Comunidad de Madrid, Madrid, Dirección General de Investigación de la Consejería de Educación y Cultura.

Torres Alberó, C. (1994): Sociología política de la ciencia, Madrid, Centro de Investigaciones Sociológicas/Siglo XXI.

Villagrá Rubio, A. (1992): «Scientific Production of Spanish Universities in the Fields of Social Sciences and Language», Scientometrics, vol. 24, n. ${ }^{\circ} 1$, pp.3-19.

ZIMAN, J. H. (1972): El conocimiento público, México, FCE (or.: 1968).

\section{ABSTRACT}

This article introduces a bibliometric study of the Revista Española de Investigaciones Sociológicas on the occasion of its twenty-fifth anniversary. The three aspects studied are production, collaboration and the subject matter handled. The importance of this journal in Spain makes this study highly representative of sociological literature published in this country. The study is likewise completed with a section dealing with its quality as a scientific journal. 


\section{TEXTO CLÁSICO}

